\title{
A Generalization of Prešić Type Mappings in Metric-Like Spaces
}

\author{
Satish Shukla ${ }^{1}$ and Brian Fisher ${ }^{2}$ \\ ${ }^{1}$ Department of Applied Mathematics, Shri Vaishnav Institute of Technology \& Science, Gram Baroli, Sanwer Road, Indore, \\ Madhya Pradesh 453331, India \\ ${ }^{2}$ Department of Mathematics, University of Leicester, Leicester LE1 7RH, UK
}

Correspondence should be addressed to Satish Shukla; satishmathematics@yahoo.co.in

Received 20 March 2013; Accepted 1 April 2013

Academic Editor: Ram U. Verma

Copyright (C) 2013 S. Shukla and B. Fisher. This is an open access article distributed under the Creative Commons Attribution License, which permits unrestricted use, distribution, and reproduction in any medium, provided the original work is properly cited.

We generalize the result of Prešić in metric-like spaces by proving some common fixed point theorems for Prešić type mappings in metric-like spaces. An example is given which shows that the generalization is proper.

\section{Introduction and Preliminaries}

Let $(X, d)$ be any metric space, and let $f: X \rightarrow X$ be any mapping; then $f$ is said to be a contraction on $X$ if there exists $\lambda \in[0,1)$ such that

$$
d(f x, f y) \leq \lambda d(x, y), \quad \forall x, y \in X .
$$

A point $x \in X$ is called a fixed point of $f$ if $f x=$ $x$. Banach [1] proved that every contraction on a complete metric space has a unique fixed point and this result is known as the Banach contraction principle. There are several generalizations of this famous principle. One such generalization is given by Prešić $[2,3]$. When studying the convergence of some particular sequences, Prešić $[2,3]$ proved the following theorem.

Theorem 1. Let $(X, d)$ be a complete metric space, $k$ a positive integer, and $f: X^{k} \rightarrow X$ a mapping satisfying the following contractive type condition:

$$
d\left(f\left(x_{1}, x_{2}, \ldots, x_{k}\right), f\left(x_{2}, x_{3}, \ldots, x_{k+1}\right)\right) \leq \sum_{i=1}^{k} q_{i} d\left(x_{i}, x_{i+1}\right),
$$

for every $x_{1}, x_{2}, \ldots, x_{k+1} \in X$, where $q_{1}, q_{2}, \ldots, q_{k}$ are nonnegative constants such that $q_{1}+q_{2}+\cdots+q_{k}<1$. Then there exists a unique point $x \in X$ such that $f(x, x, \ldots, x)=x$.
Moreover, if $x_{1}, x_{2}, \ldots, x_{k}$ are arbitrary points in $X$ and for $n \in \mathbb{N}$,

$$
x_{n+k}=f\left(x_{n}, x_{n+1}, \ldots, x_{n+k-1}\right) \text {, }
$$

then the sequence $\left\{x_{n}\right\}$ is convergent and $\lim x_{n}=$ $f\left(\lim x_{n}, \lim x_{n}, \ldots, \lim x_{n}\right)$.

Note that the $k$-step iterative sequence given by (3) represents a nonlinear difference equation. In view of Prešić theorem, it is obvious that if this sequence is convergent (which is ensured by the Prešic theorem) then the limit of the sequence is a fixed point of $f$. The result of Prešic is generalized by several authors, and some generalizations and applications of Prešić theorem can be seen in [4-15].

On the other hand, Matthews [16] introduced the notion of a partial metric space as a part of the study of denotational semantics of a dataflow network. In this space, the usual metric is replaced by a partial metric with an interesting property that the self-distance of any point of space may not be zero. Further, Matthews showed that the Banach contraction principle is valid in a partial metric space and can be applied in program verifications. O'Neill [17] generalized the concept of a partial metric space a bit further by admitting negative distances. The partial metric defined by O'Neill is called the dualistic partial metric. Heckmann [18] generalized it by omitting the small self-distance axiom. The partial metric defined by Heckmann is called a weak partial metric. 
Recently, Amini-Harandi [19] generalized the partial metric spaces by introducing the metric-like spaces. AminiHarandi introduced the notion of a $\sigma$-Cauchy sequence and completeness of metric-like spaces and proved some fixed point theorems in such spaces. In this paper, we prove some common fixed point theorems for Prešić type mappings in metric-like spaces. Our results generalize and extend the result of Prešić from complete spaces into $\sigma$-complete metriclike spaces. An example is given which shows that the generalization is proper.

First we recall some definitions about partial metric and metric-like spaces.

Definition 2 (see [16]). A partial metric on a nonempty set $X$ is a function $p: X \times X \rightarrow \mathbb{R}^{+}\left(\mathbb{R}^{+}\right.$stands for nonnegative reals) such that for all $x, y, z \in X$ :

$$
\begin{aligned}
& \text { (p1) } x=y \text { if and only if } p(x, x)=p(x, y)=p(y, y) \text {; } \\
& \text { (p2) } p(x, x) \leq p(x, y) \text {; } \\
& \text { (p3) } p(x, y)=p(y, x) \text {; } \\
& \text { (p4) } p(x, y) \leq p(x, z)+p(z, y)-p(z, z) .
\end{aligned}
$$

A partial metric space is a pair $(X, p)$ such that $X$ is a nonempty set and $p$ is a partial metric on $X$. A sequence $\left\{x_{n}\right\}$ in $(X, p)$ converges to a point $x \in X$ if and only if $p(x, x)=$ $\lim _{n \rightarrow \infty} p\left(x_{n}, x\right)$. A sequence $\left\{x_{n}\right\}$ in $(X, p)$ is called a $p$ Cauchy sequence if $\lim _{n, m \rightarrow \infty} p\left(x_{n}, x_{m}\right)$ exists and is finite. $(X, p)$ is said to be complete if every $p$-Cauchy sequence $\left\{x_{n}\right\}$ in $X$ converges to a point $x \in X$ such that $p(x, x)=$ $\lim _{n, m \rightarrow \infty} p\left(x_{n}, x_{m}\right)$.

Definition 3 (see [19]). A metric-like on a nonempty set $X$ is a function $\sigma: X \times X \rightarrow \mathbb{R}^{+}$such that for all $x, y, z \in X$ :

$$
\begin{aligned}
& (\sigma 1) \sigma(x, y)=0 \text { implies } x=y \\
& (\sigma 2) \sigma(x, y)=\sigma(y, x) \\
& (\sigma 3) \sigma(x, y) \leq \sigma(x, z)+\sigma(z, y) .
\end{aligned}
$$

A metric-like space is a pair $(X, \sigma)$ such that $X$ is a nonempty set and $\sigma$ is a metric-like on $X$. Note that a metric-like satisfies all the conditions of metric except that $\sigma(x, x)$ may be positive for some $x \in X$. Each metric-like $\sigma$ on $X$ generates a topology $\tau_{\sigma}$ on $X$ whose base is the family of open $\sigma$-balls

$$
\begin{array}{r}
B_{\sigma}(x, \epsilon)=\{y \in X:|\sigma(x, y)-\sigma(x, x)|<\epsilon\}, \\
\forall x \in X, \epsilon>0 .
\end{array}
$$

A sequence $\left\{x_{n}\right\}$ in $X$ converges to a point $x \in X$ if and only if $\lim _{n \rightarrow \infty} \sigma\left(x_{n}, x\right)=\sigma(x, x)$. A sequence $\left\{x_{n}\right\}$ is said to be $\sigma$-Cauchy if $\lim _{n, m \rightarrow \infty} \sigma\left(x_{n}, x_{m}\right)$ exists and is finite. The metric-like space $(X, \sigma)$ is called complete if for each $\sigma$ Cauchy sequence $\left\{x_{n}\right\}$, there exists $x \in X$ such that

$$
\lim _{n \rightarrow \infty} \sigma\left(x_{n}, x\right)=\sigma(x, x)=\lim _{m, n \rightarrow \infty} \sigma\left(x_{n}, x_{m}\right) .
$$

Every partial metric space is a metric-like space but the converse may not be true.
Example 4 (see [19]). Let $X=\{0,1\}$ and $\sigma: X \times X \rightarrow \mathbb{R}^{+}$be defined by

$$
\sigma(x, y)= \begin{cases}2, & \text { if } x=y=0 \\ 1, & \text { otherwise. }\end{cases}
$$

Then $(X, \sigma)$ is a metric-like space, but it is not a partial metric space, as $\sigma(0,0) \not \delta \sigma(0,1)$.

Example 5. Let $X=\mathbb{R}, k \geq 0$, and $\sigma: X \times X \rightarrow \mathbb{R}^{+}$be defined by

$$
\sigma(x, y)= \begin{cases}2 k, & \text { if } x=y=0 \\ k, & \text { otherwise }\end{cases}
$$

Then $(X, \sigma)$ is a metric-like space, but for $k>0$, it is not a partial metric space, as $\sigma(0,0) \not \sigma \sigma(0,1)$.

Example 6. Let $X=\mathbb{R}^{+}$and $\sigma: X \times X \rightarrow \mathbb{R}^{+}$be defined by

$$
\sigma(x, y)= \begin{cases}2 x, & \text { if } x=y \\ \max \{x, y\}, & \text { otherwise. }\end{cases}
$$

Then $(X, p)$ is a metric-like space, but it is not a partial metric space, as $\sigma(1,1)=2 \not \sigma(0,1)=1$.

For the following definition, we refer to [10] and the references therein.

Definition 7. Let $X$ be a nonempty set, $k$ a positive integer, and $f: X^{k} \rightarrow X$ a mapping. If $f(x, x, \ldots, x)=x$, then $x \in X$ is called a fixed point of $f$.

Definition 8 . Let $X$ be a nonempty set, $k$ a positive integer, and $f: X^{k} \rightarrow X$ and $g: X \rightarrow X$ mappings.

(a) An element $x \in X$ is said to be a coincidence point of $f$ and $g$ if $g x=f(x, \ldots, x)$.

(b) If $w=g x=f(x, \ldots, x)$, then $w$ is called a point of coincidence of $f$ and $g$.

(c) If $x=g x=f(x, \ldots, x)$, then $x$ is called a common fixed point of $f$ and $g$.

(d) Mappings $f$ and $g$ are said to be commuting if $g(f(x, \ldots, x))=f(g x, \ldots, g x)$ for all $x \in X$.

(e) Mappings $f$ and $g$ are said to be weakly compatible if they commute at their coincidence points.

Let $(X, \sigma)$ be a metric-like space, $k$ a positive integer, and $f$ : $X^{k} \rightarrow X$ a mapping. $f$ is said to be Prešić type if

$$
\sigma\left(f\left(x_{1}, x_{2}, \ldots, x_{k}\right), f\left(x_{2}, x_{3} \ldots, x_{k+1}\right)\right) \leq \sum_{i=1}^{k} \alpha_{i} \sigma\left(x_{i}, x_{i+1}\right),
$$

for all $x_{1}, x_{2}, \ldots, x_{k}, x_{k+1} \in X$, where $\alpha_{i}$ are nonnegative constants such that $\sum_{i=1}^{k} \alpha_{i}<1$.

The following lemma shows that the self-distance of a point of coincidence of two maps of a metric-like space satisfying Prešić type contractive condition is minimum (zero), and it will be used in the sequel. 
Lemma 9. Let $(X, \sigma)$ be a metric-like space, $k$ a positive integer, and $f: X^{k} \rightarrow X, g: X \rightarrow X$ two mappings. Suppose that the following condition holds:

$$
\begin{gathered}
\sigma\left(f\left(x_{1}, x_{2}, \ldots, x_{k}\right), f\left(x_{2}, x_{3}, \ldots, x_{k+1}\right)\right) \\
\leq \sum_{i=1}^{k} \alpha_{i} \sigma\left(g x_{i}, g x_{i+1}\right),
\end{gathered}
$$

for all $x_{1}, x_{2}, \ldots, x_{k+1} \in X$, where $\alpha_{1}, \alpha_{2}, \ldots, \alpha_{k}$ are nonnegative constants such that $\alpha_{1}+\alpha_{2}+\cdots+\alpha_{k}<1$. If $f$ and $g$ have a point of coincidence $v \in X$, then $\sigma(v, v)=0$.

Proof. Let $v \in X$ be any point of coincidence of $f$ and $g$. Then there exists $u \in X$ such that $f(u, u, \ldots, u)=g u=v$.

Now suppose that $\sigma(v, v) \neq 0$. Then it follows from (10) that

$$
\begin{aligned}
\sigma(v, v) & =\sigma(f(u, u, \ldots, u), f(u, u, \ldots, u)) \\
& \leq \alpha_{1} \sigma(g u, g u)+\alpha_{2} \sigma(g u, g u)+\cdots+\alpha_{k} \sigma(g u, g u) \\
& =\left[\alpha_{1}+\alpha_{2}+\cdots+\alpha_{k}\right] \sigma(g u, g u) \\
& =\left[\alpha_{1}+\alpha_{2}+\cdots+\alpha_{k}\right] \sigma(v, v) \\
& <\sigma(v, v) \quad\left(\text { as } \alpha_{1}+\alpha_{2}+\cdots+\alpha_{k}<1\right),
\end{aligned}
$$

a contradiction. Therefore we must have $\sigma(v, v)=0$.

Now we can state our main results.

\section{Main Results}

The following theorem extends and generalizes the result of Prešić in metric-like spaces.

Theorem 10. Let $(X, \sigma)$ be a metric-like space, $k$ a positive integer, and $f: X^{k} \rightarrow X, g: X \rightarrow X$ two mappings such that $f\left(X^{k}\right) \subset g(X)$ and $g(X)$ is a complete subspace of $X$. Suppose that the following condition holds:

$$
\begin{gathered}
\sigma\left(f\left(x_{1}, x_{2}, \ldots, x_{k}\right), f\left(x_{2}, x_{3}, \ldots, x_{k+1}\right)\right) \\
\leq \sum_{i=1}^{k} \alpha_{i} \sigma\left(g x_{i}, g x_{i+1}\right),
\end{gathered}
$$

for every $x_{1}, x_{2}, \ldots, x_{k+1} \in X$, where $\alpha_{1}, \alpha_{2}, \ldots, \alpha_{k}$ are nonnegative constants such that $\alpha_{1}+\alpha_{2}+\cdots+\alpha_{k}<1$. Then $f$ and $g$ have a unique point of coincidence $v \in X$ and $\sigma(v, v)=0$. Moreover, if $f$ and $g$ are weakly compatible, then $v$ is the unique common fixed point of $f$ and $g$.

Proof. Let $x_{1}, x_{2}, \ldots, x_{k} \in X$ be arbitrary points. As $f\left(X^{k}\right) \subset$ $g(X)$, we can define a sequence $\left\{y_{n}\right\}$ in $X$ by $y_{n}=g x_{n}$ for $n=1,2, \ldots, k$ and $y_{n+k}=g x_{n+k}=f\left(x_{n}, x_{n+1}, \ldots, x_{n+k-1}\right)$ for $n=1,2, \ldots$.

For simplicity put $\sigma_{n}=\sigma\left(y_{n}, y_{n+1}\right)$ for $n=1,2, \ldots$ and $\mu=\max \left\{\sigma_{1} / \theta, \sigma_{2} / \theta^{2}, \ldots, \sigma_{k} / \theta^{k}\right\}$, where $\theta=\left[\sum_{i=1}^{k} \alpha_{i}\right]^{1 / k}<1$.
By mathematical induction, we will show that

$$
\sigma_{n} \leq \mu \theta^{n} \quad \forall n \in \mathbb{N} .
$$

According to the definition of $\mu$, it is clear that (13) is true for $n=1,2, \ldots, k$. Let the following $k$ inequalities:

$$
\sigma_{n} \leq \mu \theta^{n}, \sigma_{n+1} \leq \mu \theta^{n+1}, \ldots, \sigma_{n+k-1} \leq \mu \theta^{n+k-1},
$$

be the induction hypothesis.

Now using (12), we obtain

$$
\begin{aligned}
\sigma_{n+k}= & \sigma\left(y_{n+k}, y_{n+k+1}\right) \\
= & \sigma\left(f\left(x_{n}, x_{n+1}, \ldots, x_{n+k-1}\right), f\left(x_{n+1}, x_{n+2}, \ldots, x_{n+k}\right)\right) \\
\leq & \alpha_{1} \sigma\left(g x_{n}, g x_{n+1}\right)+\alpha_{2} \sigma\left(g x_{n+1}, g x_{n+2}\right) \\
& +\cdots+\alpha_{k} \sigma\left(g x_{n+k-1}, g x_{n+k}\right) \\
= & \alpha_{1} \sigma\left(y_{n}, y_{n+1}\right)+\alpha_{2} \sigma\left(y_{n+1}, y_{n+2}\right) \\
& +\cdots+\alpha_{k} \sigma\left(y_{n+k-1}, y_{n+k}\right) \\
= & \alpha_{1} \sigma_{n}+\alpha_{2} \sigma_{n+1}+\cdots+\alpha_{k} \sigma_{n+k-1} \\
\leq & \alpha_{1} \mu \theta^{n}+\alpha_{2} \mu \theta^{n+1}+\cdots+\alpha_{k} \mu \theta^{n+k-1} \\
\leq & \alpha_{1} \mu \theta^{n}+\alpha_{2} \mu \theta^{n}+\cdots+\alpha_{k} \mu \theta^{n} \\
\quad & \quad\left(\text { as } \theta=\left[\sum_{i=1}^{k} \alpha_{i}\right]^{1 / k}<1\right) \\
= & {\left[\alpha_{1}+\alpha_{2}+\cdots+\alpha_{k}\right] \mu \theta^{n}=\left[\sum_{i=1}^{k} \alpha_{i}\right] \mu \theta^{n} } \\
= & \mu \theta^{n+k} .
\end{aligned}
$$

Thus, the inductive proof of (13) is complete.

Now let $n, m \in \mathbb{N}$ with $m>n$. Then from (13), we have

$$
\begin{aligned}
\sigma\left(y_{n}, y_{m}\right) \leq & \sigma\left(y_{n}, y_{n+1}\right)+\sigma\left(y_{n+1}, y_{n+2}\right) \\
& +\cdots+\sigma\left(y_{m-1}, y_{m}\right) \\
= & \sigma_{n}+\sigma_{n+1}+\cdots+\sigma_{m-1} \\
\leq & \mu \theta^{n}+\mu \theta^{n+1}+\mu \theta^{n+2}+\cdots \\
= & \mu \theta^{n}\left[1+\theta+\theta^{2}+\cdots\right] \\
= & \frac{\mu \theta^{n}}{1-\theta} .
\end{aligned}
$$

As, $\theta<1$, it follows from the previous inequality that

$$
\lim _{n, m \rightarrow \infty} \sigma\left(y_{n}, y_{m}\right)=0 .
$$

Thus, $\left\{y_{n}\right\}=\left\{g x_{n}\right\}$ is a $\sigma$-Cauchy sequence in $(X, \sigma)$. By the completeness of $g(X)$, there exists $u, v \in X$ such that $v=g u$ and

$$
\lim _{n \rightarrow \infty} \sigma\left(y_{n}, v\right)=\lim _{m, n \rightarrow \infty} \sigma\left(y_{n}, y_{m}\right)=\sigma(v, v)=0 .
$$


We will now show that $v$ is a point of coincidence of $f$ and g. For any $n \in \mathbb{N}$ we have

$$
\begin{aligned}
\sigma(g u, & f(u, u, \ldots, u)) \\
\leq & \sigma\left(g u, y_{n+k}\right)+\sigma\left(y_{n+k}, f(u, u, \ldots, u)\right) \\
\leq & \sigma\left(v, y_{n+k}\right)+\sigma\left(f\left(x_{n}, \ldots, x_{n+k-1}\right), f(u, u, \ldots, u)\right) \\
\leq & \sigma\left(v, y_{n+k}\right)+\sigma\left(f\left(x_{n}, \ldots, x_{n+k-1}\right)\right. \\
& \left.\quad f\left(x_{n+1}, \ldots, x_{n+k-1}, u\right)\right) \\
& +\sigma\left(f\left(x_{n+1}, \ldots, x_{n+k-1}, u\right)\right. \\
& \left.\quad f\left(x_{n+2}, \ldots, x_{n+k-1}, u, u\right)\right) \\
& +\cdots+\sigma\left(f\left(x_{n+k-1}, u, \ldots, u\right), f(u, u, \ldots, u)\right) .
\end{aligned}
$$

Using (12) and writing $\sigma_{n}=\sigma\left(y_{n}, y_{n+1}\right)=\sigma\left(g x_{n}, g x_{n+1}\right)$, $g u=v$ in the previous inequality we obtain

$$
\begin{aligned}
\sigma(v, f(u, u, \ldots, u)) \leq & \sigma\left(v, y_{n+k}\right)+\alpha_{1} \sigma_{n} \\
& +\cdots+\alpha_{k-1} \sigma_{n+k-2}+\alpha_{k} \sigma\left(y_{n+k-1}, v\right) \\
& +\alpha_{1} \sigma_{n+1}+\cdots+\alpha_{k-2} \sigma_{n+k-2} \\
& +\alpha_{k-1} \sigma\left(y_{n+k-1}, v\right)+\alpha_{k} \sigma(v, v) \\
& +\cdots+\alpha_{1} \sigma\left(y_{n+k-1}, v\right) \\
& +\left[\alpha_{2}+\cdots+\alpha_{k}\right] \sigma(v, v) .
\end{aligned}
$$

Letting $n \rightarrow \infty$ and using (18) in the previous inequality, we obtain

$$
\sigma(v, f(u, u, \ldots, u))=0, \quad \text { that is } f(u, u, \ldots, u)=v=g u \text {. }
$$

\section{Therefore, $v$ is a point of coincidence of $f$ and $g$.}

We will now show that it is unique. Suppose that $v^{\prime}$ is another point of coincidence of $f$ and $g$. Then there exists $u^{\prime} \in X$ such that $f\left(u^{\prime}, u^{\prime}, \ldots, u^{\prime}\right)=g u^{\prime}=v^{\prime}$. By Lemma 9, we have $\sigma\left(v^{\prime}, v^{\prime}\right)=0$. Now it follows from (12) that

$$
\begin{aligned}
\sigma\left(v, v^{\prime}\right)= & \sigma\left(f(u, u, \ldots, u), f\left(u^{\prime}, u^{\prime}, \ldots, u^{\prime}\right)\right) \\
\leq & \sigma\left(f(u, \ldots, u), f\left(u, \ldots, u, u^{\prime}\right)\right) \\
& +\sigma\left(f\left(u, \ldots, u, u^{\prime}\right), f\left(u, \ldots, u, u^{\prime}, u^{\prime}\right)\right) \\
& +\cdots+\sigma\left(f\left(u, u^{\prime} \ldots, u^{\prime}\right), f\left(u^{\prime}, u^{\prime}, \ldots, u^{\prime}\right)\right)
\end{aligned}
$$

$$
\begin{aligned}
\leq & \alpha_{1} \sigma(g u, g u)+\cdots+\alpha_{k-1} \sigma(g u, g u) \\
& +\alpha_{k} \sigma\left(g u, g u^{\prime}\right)+\alpha_{1} \sigma(g u, g u) \\
& +\cdots+\sigma_{k-2} \sigma(g u, g u)+\alpha_{k-1} \sigma\left(g u, g u^{\prime}\right) \\
& +\alpha_{k} \sigma\left(g u^{\prime}, g u^{\prime}\right)+\cdots+\alpha_{1} \sigma\left(g u, g u^{\prime}\right) \\
& +\alpha_{2} \sigma\left(g u^{\prime}, g u^{\prime}\right)+\cdots+\alpha_{k} \sigma\left(g u^{\prime}, g u^{\prime}\right) .
\end{aligned}
$$

As $\sigma(g u, g u)=\sigma(v, v)=0=\sigma\left(v^{\prime}, v^{\prime}\right)=\sigma\left(g u^{\prime}, g u^{\prime}\right)$, it follows from previous inequality that

$$
\begin{aligned}
\sigma\left(v, v^{\prime}\right) & \leq\left[\alpha_{1}+\alpha_{2}+\cdots+\alpha_{k}\right] p\left(g u, g u^{\prime}\right) \\
& =\left[\alpha_{1}+\alpha_{2}+\cdots+\alpha_{k}\right] p\left(v, v^{\prime}\right) \\
& <p\left(v, v^{\prime}\right) \quad\left(\text { as } \alpha_{1}+\alpha_{2}+\cdots+\alpha_{k}<1\right),
\end{aligned}
$$

a contradiction. Therefore, we must have $\sigma\left(v, v^{\prime}\right)=0$, that is, $v=v^{\prime}$. Thus the point of coincidence of $f$ and $g$ is unique.

Now suppose that $f$ and $g$ are weakly compatible. Put $w=$ $g v$. Then $w=g v=g f(u, u, \ldots, u)=f(g u, g u, \ldots, g u)=$ $f(v, v, \ldots, v)$, so $w$ is a point of coincidence of $f$ and $g$. However, by the uniqueness of $v$, we have $w=v=g v=$ $f(v, v, \ldots, v)$. Thus, $v$ is the unique common fixed point of $f$ and $g$.

Taking $f=I_{X}$ (i.e., the identity mapping of $X$ ) we obtain following fixed point result for Prešić type mapping in a metric-like space.

Corollary 11. Let $(X, \sigma)$ be a complete metric-like space, $k$ a positive integer, and $f: X^{k} \rightarrow X$ a Prešić type mapping on $X$. Then $f$ has a unique fixed point $v \in X$ and $\sigma(v, v)=0$.

The following example shows that Corollary 11 is a proper generalization of Theorem 1 .

Example 12. Let $X=[0,1]$, and define $\sigma: X \times X \rightarrow \mathbb{R}^{+}$by

$$
\sigma(x, y)= \begin{cases}2 x, & \text { if } x=y, \\ \max \{x, y\}, & \text { otherwise. }\end{cases}
$$

Then, $(X, \sigma)$ is a complete metric-like space. Note that $(X, \sigma)$ is not a partial metric space, as $\sigma(1,1) \not \sigma(0,1)$.

Define $f: X^{2} \rightarrow X$ by

$$
f(x, y)= \begin{cases}\frac{1}{16}, & \text { if } x=y=1 \\ \frac{x+y}{8}, & \text { otherwise. }\end{cases}
$$

Note that $f$ is not a Prešić type mapping in the usual metric space $(X, d)$, where $d(x, y)=|x-y|$ for all $x, y \in X$. Indeed, for the points $x_{1}=x_{2}=1, x_{3}=9 / 10$ we have

$$
d\left(f\left(x_{1}, x_{2}\right), f\left(x_{2}, x_{3}\right)\right)=d\left(\frac{1}{16}, \frac{19}{80}\right)=\frac{7}{40},
$$


and $\alpha_{1} d(1,1)+\alpha_{2} d(1,9 / 10)=(1 / 10) \alpha_{2}$. Therefore, the condition (2) of Theorem 1 is not satisfied with $\alpha_{1}+\alpha_{2}<1$. Thus the result of Prešić is not applicable here.

On the other hand, it is easy to see that $f$ satisfies all the conditions of Corollary 11, with $\alpha_{1}=\alpha_{2} \in[1 / 8,1)$ and $f(0,0)=0$; that is, 0 is the unique fixed point of $f$.

\section{References}

[1] S. Banach, "Sur les operations dans les ensembles abstraits et leur application aux equations integrales," Fundamenta Mathematicae, vol. 3, pp. 133-181, 1922.

[2] S. B. Prešić, "Sur la convergence des suites," Comptes Rendus de l'Académie des Sciences de Paris, vol. 260, pp. 3828-3830, 1965.

[3] S. B. Prešić, "Sur une classe d'inéquations aux différences finies et sur la convergence de certaines suites," Publications de l'Institut Mathématique, vol. 5 (19), pp. 75-78, 1965.

[4] K. P. R. Rao, Md. M. Ali, and B. Fisher, "Some presic type generalizations of the Banach contraction principle," Mathematica Moravica, vol. 15, pp. 41-47, 2011.

[5] L. B. C. Ćirić and S. B. Prešić, "On Prešić type generalization of the Banach contraction mapping principle," Acta Mathematica Universitatis Comenianae, vol. 76, no. 2, pp. 143-147, 2007.

[6] M. Pãcurar, "A multi-step iterative method for approximating common fixed points of Prešić-Rus type operators on metric spaces," Studia Universitatis Babes-Bolyai, Mathematica, vol. 55, no. 1, p. 149, 2010.

[7] M. Păcurar, "Approximating common fixed points of PresićKannan type operators by a multi-step iterative method," Analele Ştiinţifice ale Universităţii Ovidius Constanţa, vol. 17, no. 1, pp. 153-168, 2009.

[8] M. Păcurar, "Common fixed points for almost Presić type operators," Carpathian Journal of Mathematics, vol. 28, no. 1, pp. 117-126, 2012.

[9] M. S. Khan, M. Berzig, and B. Samet, "Some convergence results for iterative sequences of Prešić type and applications," Advances in Difference Equations, vol. 2012, article 38, 2012.

[10] R. George, K. Reshma, and R. Rajagopalan, "A generalised fixed point theorem of Prešić type in cone metric spaces and application to Markov process," Fixed Point Theory and Applications, vol. 2011, article 85, 2011.

[11] S. Shukla, R. Sen, and S. Radenović, "Set-valued Prešić type contraction in metric spaces," Analele Ştiinţifice ale Universităţii "Alexandru Ioan Cuza" din Iaşi Matematică. In press.

[12] S. Shukla and R. Sen, "Set-valued Prešić-Reich type mappings in metric spaces," Revista de la Real Academia de Ciencias Exactas, Fisicas y Naturales. Serie A, 2012.

[13] S. Shukla, S. Radenović, and S. Pantelić, "Some fixed point theorems for Prešić-Hardy-Rogers type contractions in metric spaces," Journal of Mathematics, vol. 2013, Article ID 295093, 8 pages, 2013.

[14] S. K. Malhotra, S. Shukla, and R. Sen, "A generalization of Banach contraction principle in ordered cone metric spaces," Journal of Advanced Mathematical Studies, vol. 5, no. 2, pp. 5967, 2012.

[15] Y.-Z. Chen, "A Prešić type contractive condition and its applications," Nonlinear Analysis: Theory, Methods \& Applications, vol. 71, no. 12, pp. e2012-e2017, 2009.

[16] S. G. Matthews, "Partial metric topology," in Proceedings of the 8th Summer Conference on General Topology and Applications, vol. 728, pp. 183-197, Annals of the New York Academy of Sciences, 1994.

[17] S. J. O'Neill, "Partial metrics, valuations, and domain theory," in Proceedings of the 11th Summer Conference on General Topology and Applications, vol. 806, pp. 304-315, Annals of the New York Academy of Sciences, 1996.

[18] R. Heckmann, "Approximation of metric spaces by partial metric spaces," Applied Categorical Structures, vol. 7, no. 1-2, pp. 71-83, 1999.

[19] A. Amini-Harandi, "Metric-like spaces, partial metric spaces and fixed points," Fixed Point Theory and Applications, vol. 2012, article 204, 2012. 


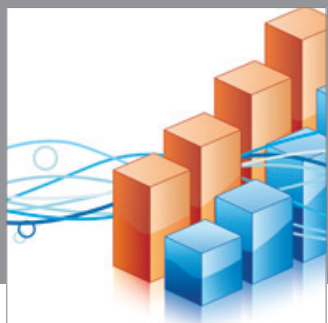

Advances in

Operations Research

mansans

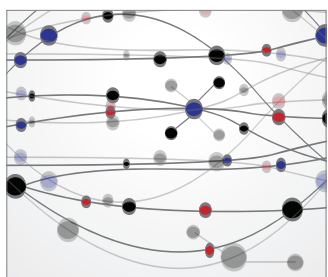

The Scientific World Journal
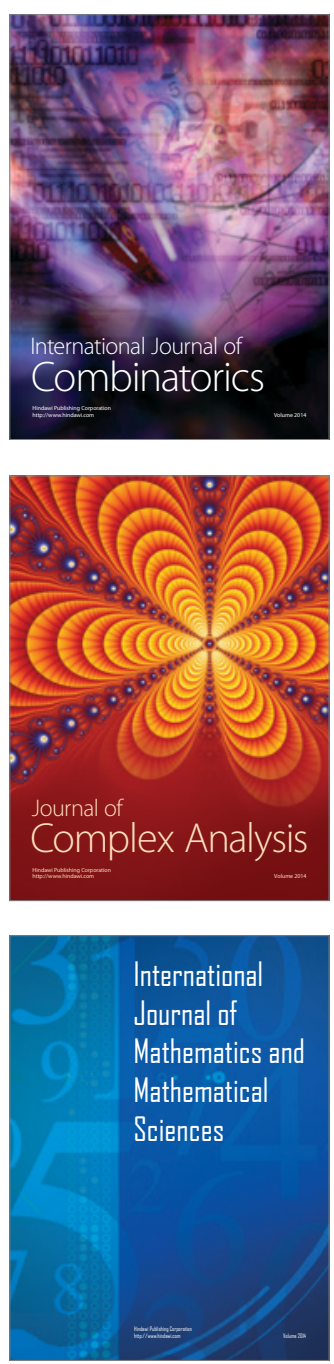
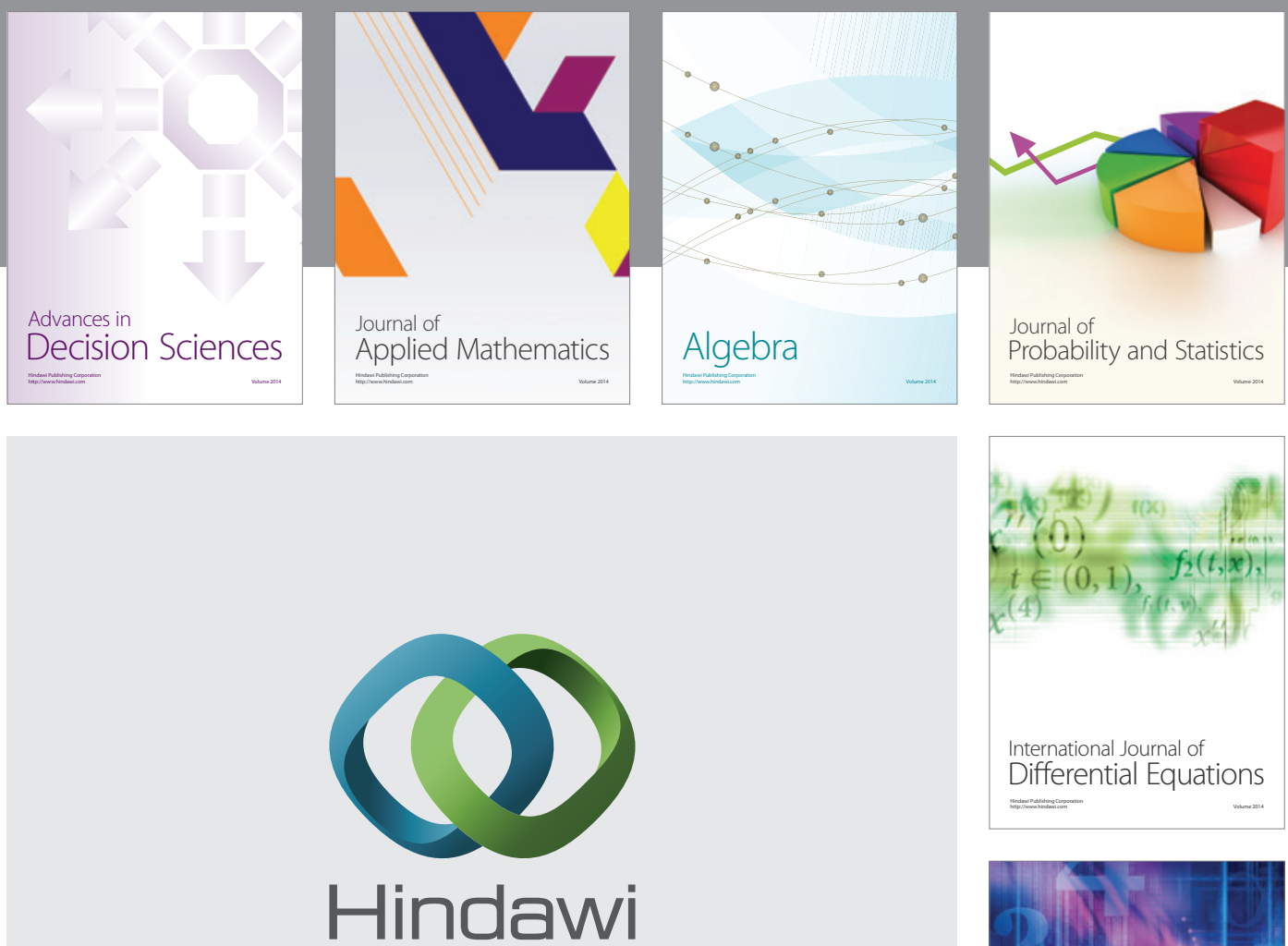

Submit your manuscripts at http://www.hindawi.com
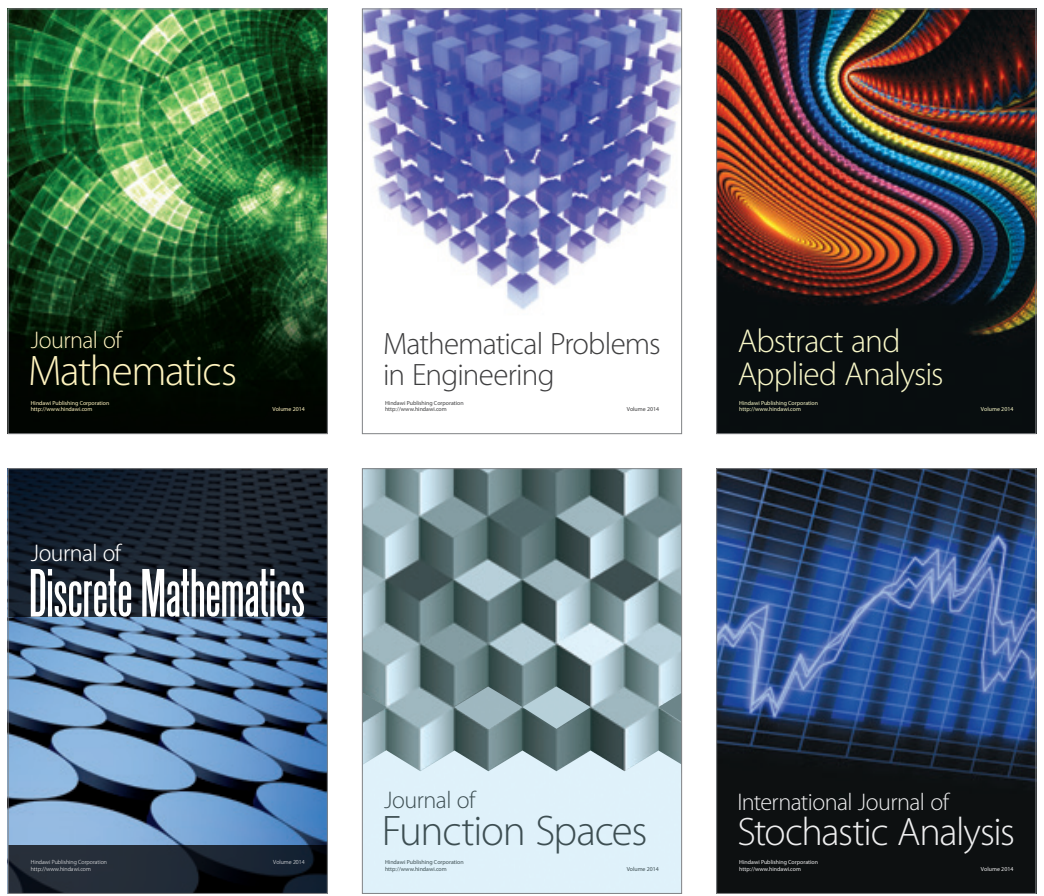

Journal of

Function Spaces

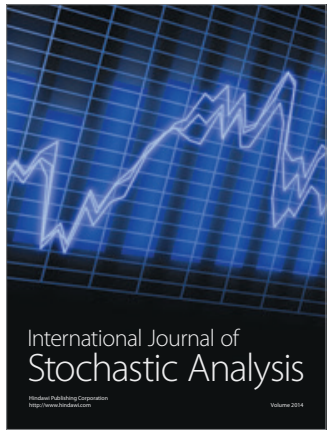

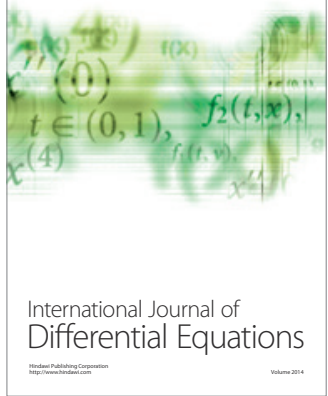
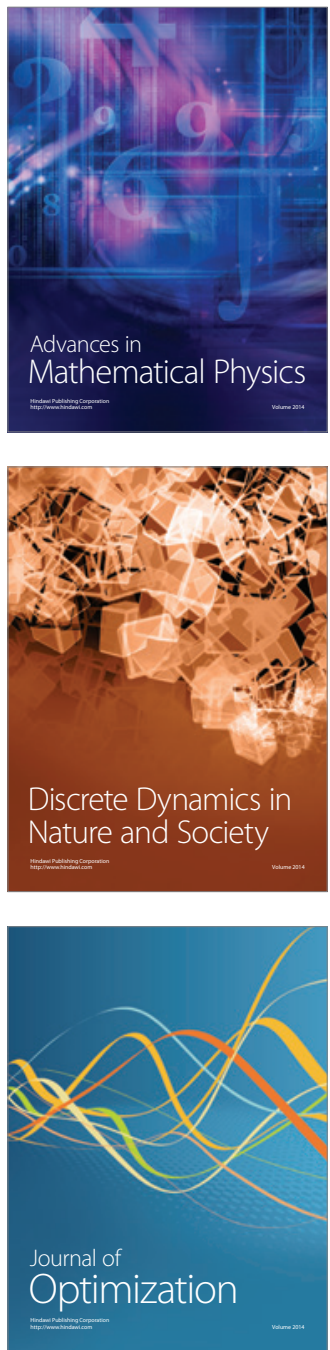